\title{
Diagnosis of miscarriages by molecular karyotyping: Benefits and pitfalls
}

\author{
Caroline Robberecht, MSc, Vicky Schuddinck, BSc, Jean-Pierre Fryns, MD, PhD, \\ and Joris Robert Vermeesch, PhD
}

\begin{abstract}
Purpose: About $50 \%$ of spontaneous abortions are caused by fetal chromosome abnormalities. Identification of these abnormalities helps to estimate recurrence risks in future pregnancies. However, due to culture failures or maternal contamination often no fetal karyotype can be obtained. Array comparative genomic hybridization can overcome some of these limitations. Methods: In this study, we analyzed 103 miscarriages by both T-banding and 1-Mb array comparative genomic hybridization. Results: We found an overall abnormality rate of $35 \%$ (34 of 96). In a comparison of 70 samples that were successfully analyzed by both techniques, 54 (77\%) had identical karyotypes (42 normal, 12 abnormal) and $16(23 \%)$ cases showed discrepancies. Most of these differences were due to maternal contamination during cell culture, which resulted erroneously in a normal female karyotype. Conclusion: These results demonstrate the improved diagnostic yield of array comparative genomic hybridization as compared with conventional karyotyping. Therefore, we implemented this technique in the diagnostic workup of miscarriages. Genet Med 2009:11(9):646-654.
\end{abstract}

Key Words: miscarriage, array comparative genomic hybridization, array $C G H$, chromosomal imbalance

Spontaneous abortions are common, with $10 \%$ to $15 \%$ of all $\int$ clinically recognized pregnancies ending in early pregnancy loss. Cytogenetic analysis has shown that about $50 \%$ of first trimester miscarriages are caused by fetal chromosome abnormalities, most of which consist of numerical abnormalities (86\%), including trisomies, monosomies, and polyploidies. Structural abnormalities represent another $6 \%$ of anomalies found. ${ }^{1-3}$ Identification of the cause of a spontaneous abortion helps to estimate recurrence risks in future pregnancies and, when an anomaly is found, comforts parents. Over the years, routine analysis of products of conception (POC) has been performed by karyotyping of metaphase spreads after tissue culture. However, due to failure of culture growth, suboptimal chromosome preparations, or possible maternal contamination ${ }^{4,5}$ either no result or an erroneous result is obtained.

New molecular cytogenetic methods avoid some of these pitfalls. Interphase fluorescence in situ hybridization (FISH), quantitative fluorescence polymerase chain reaction, and subtelomeric multiplex ligation-dependent probe amplification are rapid techniques that do not require cell culture and can be

From the Center for Human Genetics, University Hospital Gasthuisberg, Leuven, Belgium.

Joris Robert Vermeesch, Center for Human Genetics, Herestraat 49, Leuven 3000, Belgium. E-mail: Joris.Vermeesch@uz.kuleuven.ac.be.

Disclosure: The authors declare no conflict of interest

Submitted for publication November 17, 2008

Accepted for publication April 27, 2009.

Published online ahead of print July 16, 2009

DOI: 10.1097/GIM.0b013e3181abc92a performed in 24 to 48 hours. ${ }^{6-9}$ Their main disadvantage, however, is the use of probes and primers that target only a selection of chromosomes or only specific subtelomeric loci, thereby missing information about the remaining genome.

Comparative genomic hybridization (CGH) uses DNA isolated directly from the fetus or extra embryonic tissues and, after labeling with fluorescent dyes, hybridizes this DNA together with a labeled reference sample to normal metaphase spreads. ${ }^{10,11}$ It has been applied successfully by several groups to detect fetal chromosome abnormalities in POC. ${ }^{5,12-17}$ Array $\mathrm{CGH}$ takes this technique one step further by hybridizing labeled patient and reference DNA to a selection of genomic clones on a slide. ${ }^{18,19}$ The use of these clones as target DNA increases the resolution beyond the $3-\mathrm{Mb}$ limit of G-banding and especially beyond the $10-20-\mathrm{Mb}$ low-resolution banding usually applied in the study of miscarriages. Studies by Schaeffer et al., ${ }^{20}$ Benkhalifa et al., ${ }^{21}$ and Shimokawa et al. ${ }^{22}$ have shown that it is now possible to detect submicroscopic variants that were previously not detected.

In this study, we analyzed 103 POC by $1-M b$ array CGH and demonstrate an improved diagnostic yield as compared with conventional karyotyping. We implemented this technique in the diagnostic workup of miscarriages.

\section{MATERIALS AND METHODS}

\section{Sample preparation}

Samples from 103 spontaneous abortions were collected during the curettage procedure. These tissue samples were mainly placental villi, sometimes with membranes or fetal tissue. After removal of AmnioMAX medium (GIBCO Invitrogen, Paisley, UK), samples were washed with Dulbecco's Phosphate Buffered Saline (DPBS) and separated from blood clots and bloody tissue. For the first 36 cases, after again washing with DPBS, tissue was split into three fractions: one third for cell culture and subsequent karyotyping, one third for FISH analysis, and one third for DNA extraction and subsequent array $\mathrm{CGH}$ analysis. The separate fractions were then dissected into smaller pieces and put in a solution of collagenase and AmnioMAX for incubation overnight. For the remaining 67 cases, tissue was washed with DPBS, dissected, and incubated overnight in AmnioMAX medium with collagenase. This created a more homogeneous tissue mixture, in the hope of detecting more mosaic cases and more males and aberrations contaminated by maternal cells by array CGH. This mixture was then separated into three fractions as described earlier. After centrifugation, cells were sent for DNA extraction with the Autopure LS (Qiagen, Valencia, CA) or for FISH, or cells were resuspended in 5-mL AmnioMAX medium for culture growth.

\section{Karyotyping}

If growth was present, cells were analyzed using a standard T-banding protocol. ${ }^{23}$ T-banding has the advantage that subtelomeric regions are better visualized compared with G-banding. Per sample, 10 mitoses were karyotyped and 2 were analyzed in detail. 
Table 1 Karyotype and array CGH results of 103 products of conception

\begin{tabular}{|c|c|c|c|c|c|}
\hline & $\begin{array}{l}\text { Conventional } \\
\text { karyotype }\end{array}$ & Array CGH results & FISH probes & FISH results & $\begin{array}{c}\text { Proposed karyotype based } \\
\text { on array CGH and FISH } \\
\text { interpretation }\end{array}$ \\
\hline 1 & $46, X X$ & arr cgh $1-22(3348) \times 2, \mathrm{X}(155) \times 2, \mathrm{Y}(31) \times 0$ & & & $46, \mathrm{XX}$ \\
\hline 2 & $45, \mathrm{X}$ & $\operatorname{arr} \operatorname{cgh} \mathrm{X}(155) \times 1, \mathrm{Y}(31) \times 0$ & & & $45, \mathrm{X}$ \\
\hline 3 & $47, \mathrm{X},+21,+\operatorname{mar}$ & $\operatorname{arr} \operatorname{cgh} \mathrm{X}(155) \times 1, \mathrm{Y}(31) \times 0,21(36) \times 3$ & & & $46, X,+21$ \\
\hline 4 & NG & arr cgh $1-22(3348) \times 2, \mathrm{X}(155) \times 2, \mathrm{Y}(31) \times 0$ & & & $46, X X$ \\
\hline 5 & $46, X Y$ & arr cgh $1-22(3348) \times 2, \mathrm{X}(155) \times 1, \mathrm{Y}(31) \times 1$ & & & $46, X Y$ \\
\hline 6 & $46, X X$ & arr cgh $1-22(3348) \times 2, \mathrm{X}(155) \times 2, \mathrm{Y}(31) \times 0$ & & & $46, \mathrm{XX}$ \\
\hline 7 & NG & arr cgh $1-22(3348) \times 2, \mathrm{X}(155) \times 2, \mathrm{Y}(31) \times 0$ & & & $46, X X$ \\
\hline 8 & $46, X X$ & arr cgh $1-22(3348) \times 2, \mathrm{X}(155) \times 2, \mathrm{Y}(31) \times 0$ & & & $46, \mathrm{XX}$ \\
\hline 9 & $46, X Y$ & arr cgh $1-22(3348) \times 2, \mathrm{X}(155) \times 1, \mathrm{Y}(31) \times 1$ & & & $46, X Y$ \\
\hline 10 & $46, X X$ & arr cgh $1-22(3348) \times 2, \mathrm{X}(155) \times 2, \mathrm{Y}(31) \times 0$ & & & $46, \mathrm{XX}$ \\
\hline 11 & NG & arr cgh $1-22(3348) \times 2, \mathrm{X}(155) \times 2, \mathrm{Y}(31) \times 0$ & & & $46, X X$ \\
\hline 12 & NG & arr cgh $1-22(3348) \times 2, \mathrm{X}(155) \times 2, \mathrm{Y}(31) \times 0$ & & & $46, \mathrm{XX}$ \\
\hline 13 & $46, \mathrm{XX}$ & arr cgh $1-22(3348) \times 2, \mathrm{X}(155) \times 2, \mathrm{Y}(31) \times 0$ & & & $46, \mathrm{XX}$ \\
\hline 14 & $46, \mathrm{XX}$ & arr cgh $1-22(3348) \times 2, \mathrm{X}(155) \times 2, \mathrm{Y}(31) \times 0$ & & & $46, \mathrm{XX}$ \\
\hline 15 & $47, \mathrm{XY},+21$ & $\operatorname{arr} \operatorname{cgh} 21(36) \times 3$ & & & $47, \mathrm{XY},+21$ \\
\hline 16 & $47, \mathrm{XY},+13$ & arr cgh $13(106) \times 2.2$ & & & $46, X Y / 47, X Y,+13(20 \%)$ \\
\hline 17 & $46, X X$ & arr cgh $\mathrm{X}(155) \times 1.54, \mathrm{Y}(31) \times 1.39$ & $\mathrm{XY}$ & $5 \%$ male & $46, X X / 46, X Y^{a}$ \\
\hline 18 & NG & arr cgh $1-22(3348) \times 2, \mathrm{X}(155) \times 2, \mathrm{Y}(31) \times 0$ & & & $46, X X$ \\
\hline 19 & NG & $\operatorname{arr} \operatorname{cgh} 22(59) \times 3$ & & & $47, \mathrm{XX},+22$ \\
\hline 20 & $46, \mathrm{XX}$ & arr cgh $1-22(3348) \times 2, \mathrm{X}(155) \times 2, \mathrm{Y}(31) \times 0$ & & & $46, \mathrm{XX}$ \\
\hline 21 & $46, \mathrm{XX}$ & arr cgh $\mathrm{X}(155) \times 1.9, \mathrm{Y}(31) \times 0$ & $\mathrm{XY}$ & No pellet & $45, \mathrm{X}(10 \%) / 46, \mathrm{XX}^{a}$ \\
\hline 22 & $46, X X$ & arr cgh $1-22(3348) \times 2, \mathrm{X}(155) \times 2, \mathrm{Y}(31) \times 0$ & & & $46, X X$ \\
\hline 23 & $45, \mathrm{X}[4] / 46, \mathrm{XX}[6]$ & $\operatorname{arr} \operatorname{cgh} \mathrm{X}(155) \times 1.9, \mathrm{Y}(31) \times 0$ & $\mathrm{XY}$ & No pellet & $45, \mathrm{X}(10 \%) / 46, \mathrm{XX}^{a}$ \\
\hline 24 & $47, \mathrm{XY},+8$ & $\operatorname{arr} \operatorname{cgh} 8(174) \times 3$ & & & $47, \mathrm{XY},+8$ \\
\hline 25 & $46, X X$ & arr cgh $1-22(3348) \times 2, \mathrm{X}(155) \times 2, \mathrm{Y}(31) \times 0$ & & & $46, \mathrm{XX}$ \\
\hline 26 & NG & arr cgh $1-22(3348) \times 2, \mathrm{X}(155) \times 1, \mathrm{Y}(31) \times 1$ & & & $46, X Y$ \\
\hline 27 & NG & arr cgh $1-22(3348) \times 2, \mathrm{X}(155) \times 2, \mathrm{Y}(31) \times 0$ & & & $46, X X$ \\
\hline 28 & $46, X X$ & arr cgh $1-22(3348) \times 2, \mathrm{X}(155) \times 2, \mathrm{Y}(31) \times 0$ & & & $46, \mathrm{XX}$ \\
\hline 29 & $47, \mathrm{XY},+15$ & $\operatorname{arr} \operatorname{cgh} 15(96) \times 3$ & & & $47, \mathrm{XY},+15$ \\
\hline 30 & $46, \mathrm{XX}$ & arr cgh $1-22(3348) \times 2, \mathrm{X}(155) \times 2, \mathrm{Y}(31) \times 0$ & & & $46, \mathrm{XX}$ \\
\hline 31 & $45, X[4] / 46, X X[6]$ & $\operatorname{arr} \operatorname{cgh} \mathrm{X}(155) \times 1, \mathrm{Y}(31) \times 0$ & $\mathrm{XY}$ & $94 \%$ turner & $45, \mathrm{X}$ \\
\hline 32 & $46, \mathrm{XY}[3] / 46, \mathrm{XX}[11]$ & arr cgh $1-22(3348) \times 2, X(155) \times 2, Y(31) \times 0$ & $\mathrm{XY}$ & $22 \%$ male & $46, \mathrm{XX} / 46, \mathrm{XY}^{a}$ \\
\hline 33 & NG & $\operatorname{arr} \operatorname{cgh} \mathrm{X}(155) \times 2.5$ & $\mathrm{XY}$ & Insufficient material & $46, \mathrm{XX} / 47, \mathrm{XXX}^{a}$ \\
\hline 34 & $69, \mathrm{XXX}$ & arr cgh $1-22(3348) \times 2, \mathrm{X}(155) \times 2, \mathrm{Y}(31) \times 0$ & & & $46, X X$ \\
\hline 35 & $47, \mathrm{XX},+16$ & $\operatorname{arr} \operatorname{cgh} 16(111) \times 3$ & & & $47, \mathrm{XX},+16$ \\
\hline 36 & $46, \mathrm{XX}$ & $\operatorname{arr} \operatorname{cgh} \mathrm{X}(155) \times 1.84, \mathrm{Y}(31) \times 0.92$ & $\mathrm{XY}$ & $36.5 \% \mathrm{XXYY}$ & $46, \mathrm{XX} / 92, \mathrm{XXYY}^{a}$ \\
\hline 37 & $46, \mathrm{XX}$ & arr cgh $1-22(3348) \times 2, \mathrm{X}(155) \times 2, \mathrm{Y}(31) \times 0$ & & & $46, \mathrm{XX}$ \\
\hline 38 & $46, X X$ & $-^{b}$ & & & - \\
\hline 39 & $93, \mathrm{XXYY},+22$ & $\operatorname{arr} \operatorname{cgh} 22(59) \times 3$ & $\mathrm{XY}, 22$ & $\begin{array}{c}47, \mathrm{XX},+22(60 \%) / \\
46, \mathrm{XX}(40 \%)\end{array}$ & $47, \mathrm{XX},+22$ \\
\hline
\end{tabular}

(Continued) 
Table 1 Continued

\begin{tabular}{|c|c|c|c|c|c|}
\hline & $\begin{array}{l}\text { Conventional } \\
\text { karyotype }\end{array}$ & Array CGH results & FISH probes & FISH results & $\begin{array}{c}\text { Proposed karyotype based } \\
\text { on array CGH and FISH } \\
\text { interpretation }\end{array}$ \\
\hline 40 & $46, \mathrm{XX}$ & $\operatorname{arr} \operatorname{cgh} \mathrm{X}(155) \times 1.90, \mathrm{Y}(31) \times 0.69$ & $\mathrm{XY}$ & $17 \% \mathrm{XXY}$ & $46, \mathrm{XX} / 69, \mathrm{XXY}^{a}$ \\
\hline 41 & $46, X X$ & arr cgh $1-22(3348) \times 2, \mathrm{X}(155) \times 2, \mathrm{Y}(31) \times 0$ & & & $46, X X$ \\
\hline 42 & $46, \mathrm{XX}$ & arr cgh $1-22(3348) \times 2, \mathrm{X}(155) \times 2, \mathrm{Y}(31) \times 0$ & & & $46, X X$ \\
\hline 43 & NG & - & & & - \\
\hline 44 & $47, \mathrm{XX},+16$ & arr cgh $16(111) \times 3$ & & & $47, \mathrm{XX},+16$ \\
\hline 45 & $69, \mathrm{XXY}$ & - & & & - \\
\hline 46 & $46, \mathrm{XX}$ & arr cgh $1-22(3348) \times 2, \mathrm{X}(155) \times 2, \mathrm{Y}(31) \times 0$ & & & $46, X X$ \\
\hline \multirow[t]{2}{*}{47} & $46, \mathrm{XX}$ & arr cgh Xp22.31 & STS & $\begin{array}{r}100 \% 1 \text { sign } \\
\text { STS locus }\end{array}$ & 46,XXdel $(\mathrm{X})(\mathrm{p} 22.3)$ \\
\hline & & $(\mathrm{RP} 11-483 \mathrm{M} 24->\mathrm{RP} 11-143 \mathrm{E} 20) \times 1$ & & & \\
\hline 48 & $46, \mathrm{XX}$ & arr cgh $1-22(3348) \times 2, \mathrm{X}(155) \times 2, \mathrm{Y}(31) \times 0$ & & & $46, \mathrm{XX}$ \\
\hline 49 & $\begin{array}{c}46, X X, t(1 p ; 3 p) \\
(p 36.13 ; p 23)\end{array}$ & arr cgh $1-22(3348) \times 2, \mathrm{X}(155) \times 2, \mathrm{Y}(31) \times 0$ & & & $46, X X$ \\
\hline 50 & $46, \mathrm{XX}$ & arr cgh $1-22(3348) \times 2, \mathrm{X}(155) \times 2, \mathrm{Y}(31) \times 0$ & & & $46, X X$ \\
\hline 51 & $46, X X$ & arr cgh $1-22(3348) \times 2, \mathrm{X}(155) \times 2, \mathrm{Y}(31) \times 0$ & & & $46, X X$ \\
\hline 52 & $69, \mathrm{XXY}$ & arr cgh $\mathrm{X}(155) \times 1.55, \mathrm{Y}(31) \times 1.59$ & & & $69, \mathrm{XXY}$ \\
\hline 53 & $46, \mathrm{XX}$ & arr cgh $1-22(3348) \times 2, \mathrm{X}(155) \times 2, \mathrm{Y}(31) \times 0$ & & & $46, X X$ \\
\hline 54 & $\begin{array}{l}47, \mathrm{XX},+22[8] / 46 \\
\quad \mathrm{XX}[2]\end{array}$ & $\operatorname{arr} \operatorname{cgh} 22(59) \times 3$ & & & $47, \mathrm{XX},+22$ \\
\hline 55 & $45, \mathrm{X}$ & $\operatorname{arr} \operatorname{cgh} \mathrm{X}(155) \times 1.7, \mathrm{Y}(31) \times 0$ & $\mathrm{X}$ & $43.5 \%$ turner & $45, \mathrm{X}(30 \%) / 46, \mathrm{XX}^{a}$ \\
\hline 56 & $46, \mathrm{XX}$ & arr cgh $1-22(3348) \times 2, \mathrm{X}(155) \times 2, \mathrm{Y}(31) \times 0$ & & & $46, X X$ \\
\hline 57 & NG & arr cgh $1-22(3348) \times 2, \mathrm{X}(155) \times 2, \mathrm{Y}(31) \times 0$ & & & $46, X X$ \\
\hline 58 & NG & - & & & - \\
\hline 59 & NG & - & & & - \\
\hline 60 & $46, \mathrm{XX}$ & arr $\operatorname{cgh} \mathrm{X}(155) \times 1.5, \mathrm{Y}(31) \times 0$ & $\mathrm{X}$ & $42 \%$ turner & $45, \mathrm{X}(50 \%) / 46, \mathrm{XX}^{a}$ \\
\hline 61 & NG & arr cgh $1-22(3348) \times 2, \mathrm{X}(155) \times 2, \mathrm{Y}(31) \times 0$ & & & $46, X X$ \\
\hline 62 & NG & arr cgh $\mathrm{X}(155) \times 1.59, \mathrm{Y}(31) \times 1.49$ & $\mathrm{XY}, 22,13$ & $40 \% \mathrm{XXYY}$ & $46, \mathrm{XX} / 92, \mathrm{XXYY} \mathrm{Y}^{a}$ \\
\hline 63 & $47, \mathrm{XY},+13$ & - & & & - \\
\hline 64 & $46, X Y, i(7 p)$ & $\begin{array}{l}\text { arr cgh } 7 \mathrm{p}(\mathrm{CTB}-164 \mathrm{D} 18->\mathrm{RP} 5-905 \mathrm{H} 07) \times 2.2 \\
7 \mathrm{q}(\mathrm{RP} 11-797 \mathrm{H} 07->\mathrm{RP} 4-764012) \times 1.8\end{array}$ & $X Y, 7 p$ & $\begin{array}{c}46, \mathrm{XY}, \mathrm{i}(7 \mathrm{p})(40 \%) / \\
46, \mathrm{XY}(60 \%)\end{array}$ & $46, X Y, i(7 p)(20 \%) / 46, X Y$ \\
\hline 65 & NG & arr $\operatorname{cgh} X(155) \times 3,21(36) \times 3$ & $\mathrm{XY}, 21$ & ND & $48, \mathrm{XXX},+21$ \\
\hline 66 & $46, X X$ & arr cgh $1-22(3348) \times 2, \mathrm{X}(155) \times 2, \mathrm{Y}(31) \times 0$ & & & $46, X X$ \\
\hline 67 & $46, \mathrm{XX}$ & arr cgh $1-22(3348) \times 2, \mathrm{X}(155) \times 2, \mathrm{Y}(31) \times 0$ & & & $46, \mathrm{XX}$ \\
\hline 68 & $46, \mathrm{XX}$ & arr cgh $1-22(3348) \times 2, \mathrm{X}(155) \times 2, \mathrm{Y}(31) \times 0$ & & & $46, \mathrm{XX}$ \\
\hline 69 & $46, X Y$ & arr cgh $1-22(3348) \times 2, \mathrm{X}(155) \times 1, \mathrm{Y}(31) \times 1$ & & & $46, X Y$ \\
\hline 70 & $46, \mathrm{XX}$ & arr cgh $\mathrm{X}(155) \times 1.87, \mathrm{Y}(31) \times 0.79$ & $\mathrm{XY}$ & $20.5 \%$ male & $46, \mathrm{XX} / 46, \mathrm{XY}^{a}$ \\
\hline 71 & $46, \mathrm{XX}$ & - & & & - \\
\hline 72 & $45, \mathrm{X}$ & - & & & - \\
\hline 73 & $46, \mathrm{XX}$ & arr cgh $1-22(3348) \times 2, \mathrm{X}(155) \times 2, \mathrm{Y}(31) \times 0$ & & & $46, X X$ \\
\hline & & & & & (Continued) \\
\hline
\end{tabular}


Table 1 Continued

\begin{tabular}{|c|c|c|c|c|c|}
\hline & $\begin{array}{l}\text { Conventional } \\
\text { karyotype }\end{array}$ & Array CGH results & FISH probes & FISH results & $\begin{array}{l}\text { Proposed karyotype based } \\
\text { on array CGH and FISH } \\
\text { interpretation }\end{array}$ \\
\hline 74 & $46, \mathrm{XX}$ & arr cgh $1-22(3348) \times 2, X(155) \times 2, Y(31) \times 0$ & & & $46, X X$ \\
\hline 75 & $46, X X$ & arr cgh $1-22(3348) \times 2, X(155) \times 2, Y(31) \times 0$ & & & $46, \mathrm{XX}$ \\
\hline 76 & $46, \mathrm{XX}$ & arr cgh $1-22(3348) \times 2, X(155) \times 2, Y(31) \times 0$ & & & $46, \mathrm{XX}$ \\
\hline 77 & NG & arr cgh $1-22(3348) \times 2, X(155) \times 2, Y(31) \times 0$ & & & $46, \mathrm{XX}$ \\
\hline 78 & $46, X X$ & - & & & - \\
\hline 79 & $46, \mathrm{XX}$ & arr cgh $1-22(3348) \times 2, X(155) \times 2, Y(31) \times 0$ & & & $46, \mathrm{XX}$ \\
\hline 80 & $69, \mathrm{XXY}$ & - & & & - \\
\hline 81 & $46, \mathrm{XX}$ & arr cgh $1-22(3348) \times 2, X(155) \times 2, Y(31) \times 0$ & & & $46, X X$ \\
\hline 82 & NG & arr cgh $1-22(3348) \times 2, X(155) \times 1, Y(31) \times 1$ & & & $46, X Y$ \\
\hline 83 & NG & $\operatorname{arr} \operatorname{cgh} 22(59) \times 2.1$ & $\mathrm{XY}, 22$ & $\begin{array}{c}47, \mathrm{XX},+22(18 \%) / \\
46, \mathrm{XX}(82 \%)\end{array}$ & $47, \mathrm{XX},+22(10 \%) / 46, \mathrm{XX}^{a}$ \\
\hline 84 & $46, X X$ & arr cgh $1-22(3348) \times 2, X(155) \times 2, Y(31) \times 0$ & & & $46, X X$ \\
\hline 85 & $46, \mathrm{XX}$ & arr cgh $1-22(3348) \times 2, \mathrm{X}(155) \times 2, \mathrm{Y}(31) \times 0$ & & & $46, \mathrm{XX}$ \\
\hline 86 & $46, \mathrm{XX}$ & arr cgh $1-22(3348) \times 2, X(155) \times 2, Y(31) \times 0$ & & & $46, \mathrm{XX}$ \\
\hline 87 & $46, X X$ & arr $\operatorname{cgh} \mathrm{X}(155) \times 1.94, \mathrm{Y}(31) \times 0.68$ & $\mathrm{XY}$ & $7 \%$ male & $46, \mathrm{XX} / 46, \mathrm{XY}^{a}$ \\
\hline 88 & NG & $\operatorname{arr} \operatorname{cgh} 5(208) \times 1.5$ & $\mathrm{XY}, 5$ & $\begin{array}{c}91, \mathrm{XXXX},-5(30 \%) / \\
92, \mathrm{XXXX}(47 \%) / \\
46, \mathrm{XX}(23 \%)\end{array}$ & $91, \mathrm{XXXX},-5 / 92, \mathrm{XXXX} / 46, \mathrm{XX}^{a}$ \\
\hline 89 & NG & - & & & - \\
\hline 90 & $46, \mathrm{XX}$ & arr cgh $1-22(3348) \times 2, \mathrm{X}(155) \times 2, \mathrm{Y}(31) \times 0$ & & & $46, \mathrm{XX}$ \\
\hline 91 & $46, \mathrm{XX}$ & arr cgh $1-22(3348) \times 2, X(155) \times 2, Y(31) \times 0$ & & & $46, \mathrm{XX}$ \\
\hline 92 & $46, \mathrm{XX}$ & $\operatorname{arr} \operatorname{cgh} \mathrm{X}(155) \times 1.85, \mathrm{Y}(31) \times 0$ & $\mathrm{XY}$ & $46, \mathrm{XX}$ & $45, \mathrm{X}(15 \%) / 46, \mathrm{XX}^{a}$ \\
\hline 93 & $46, \mathrm{XX}$ & arr cgh $1-22(3348) \times 2, X(155) \times 2, Y(31) \times 0$ & & & $46, \mathrm{XX}$ \\
\hline 94 & NG & $\operatorname{arr} \operatorname{cgh} 21(36) \times 3$ & & & $47, \mathrm{XY},+21$ \\
\hline 95 & $46, \mathrm{XX}$ & arr cgh $1-22(3348) \times 2, X(155) \times 2, Y(31) \times 0$ & & & $46, X X$ \\
\hline 96 & NG & arr cgh $1-22(3348) \times 2, X(155) \times 2, Y(31) \times 0$ & & & $46, \mathrm{XX}$ \\
\hline 97 & NG & $\operatorname{arr} \operatorname{cgh} 13(106) \times 3$ & $\mathrm{XY}, 13$ & $\begin{array}{c}47, \mathrm{XX},+13(92 \%) / \\
46, \mathrm{XX}(8 \%)\end{array}$ & $47, \mathrm{XX},+13$ \\
\hline 98 & NG & arr cgh $1-22(3348) \times 2, X(155) \times 2, Y(31) \times 0$ & & & $46, \mathrm{XX}$ \\
\hline 99 & $46, \mathrm{XX}$ & arr cgh $1-22(3348) \times 2, X(155) \times 2, Y(31) \times 0$ & & & $46, \mathrm{XX}$ \\
\hline 100 & $46, X Y$ & arr cgh $1-22(3348) \times 2, \mathrm{X}(155) \times 1, \mathrm{Y}(31) \times 1$ & & & $46, X Y$ \\
\hline 101 & $46, X X$ & arr cgh $1-22(3348) \times 2, X(155) \times 2, Y(31) \times 0$ & & & $46, \mathrm{XX}$ \\
\hline 102 & $\begin{array}{c}\text { 46,XX,t(2;7) } \\
\text { (p10;q10) }\end{array}$ & arr cgh $1-22(3348) \times 2, X(155) \times 2, Y(31) \times 0$ & & & $46, \mathrm{XX}$ \\
\hline 103 & NG & - & & & - \\
\hline
\end{tabular}

\section{Array CGH}

Samples were analyzed by array CGH on a custom-made bacterial artificial chromosome (BAC) array with 3534 spotted clones. A total of $150 \mathrm{ng}$ patient DNA and XXY-reference ${ }^{24}$
DNA were labeled with either Cy5 dCTP or Cy3 dCTP (GE Healthcare, Chalfont St. Giles, UK) using the BioPrime ${ }^{(\mathbb{B}}$ Array CGH Genomic Labeling System (Invitrogen). All experiments were performed in double with reverse labeling of the samples. 
A

$45, \times(30 \%) / 46, \times \times(70 \%)$

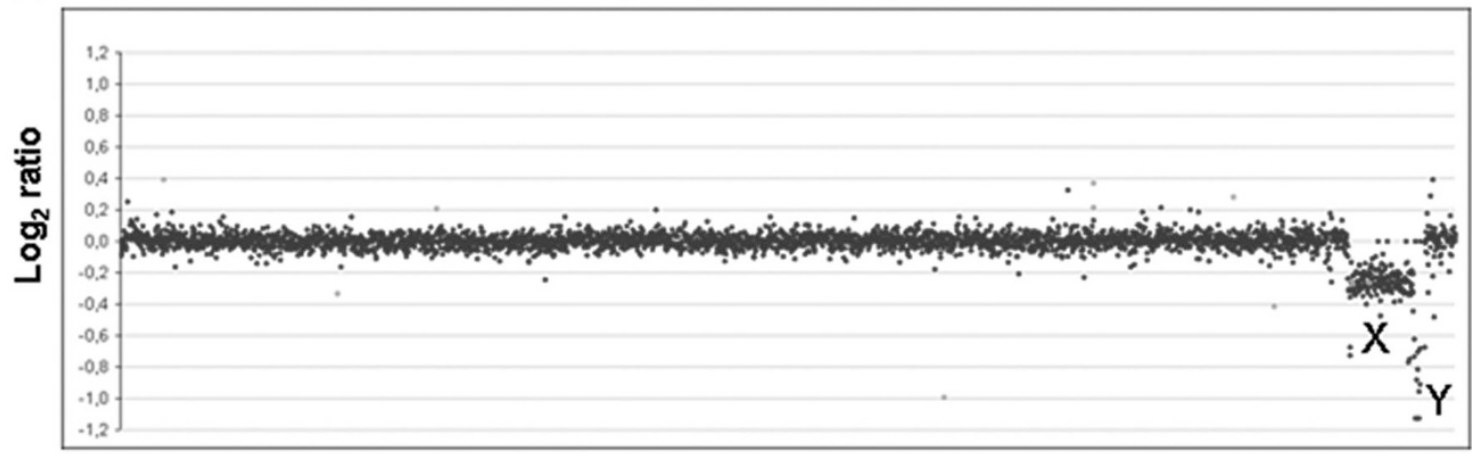

B $46, X Y(7 \%) / 46, X \times(93 \%)$

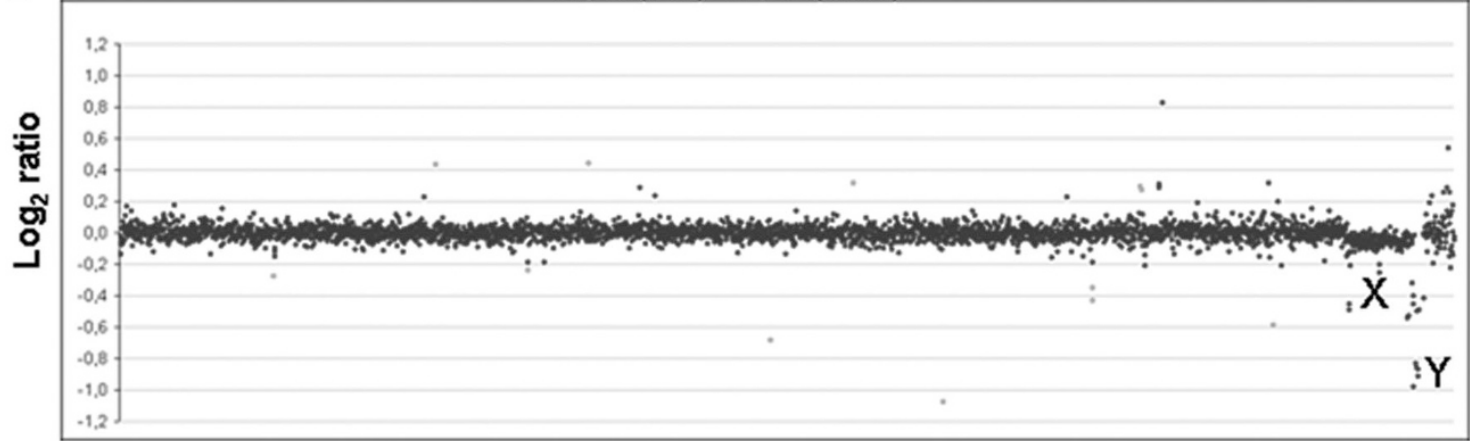

C $46, X Y, i(7 p)(20 \%) / 46, X Y(80 \%)$

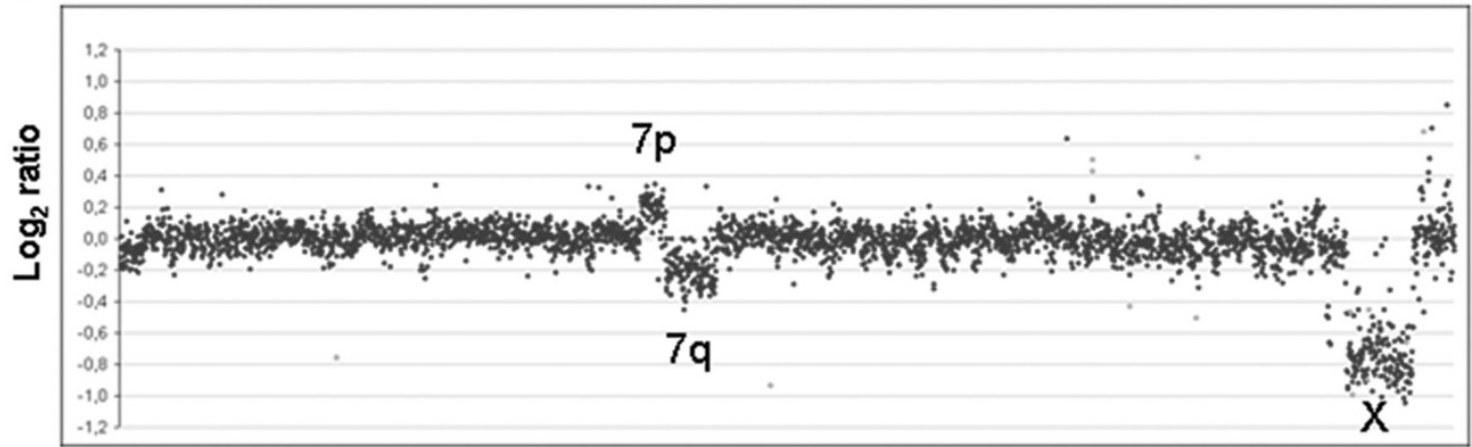

D

$47, \times X,+22(10 \%) / 46, X \times(90 \%)$

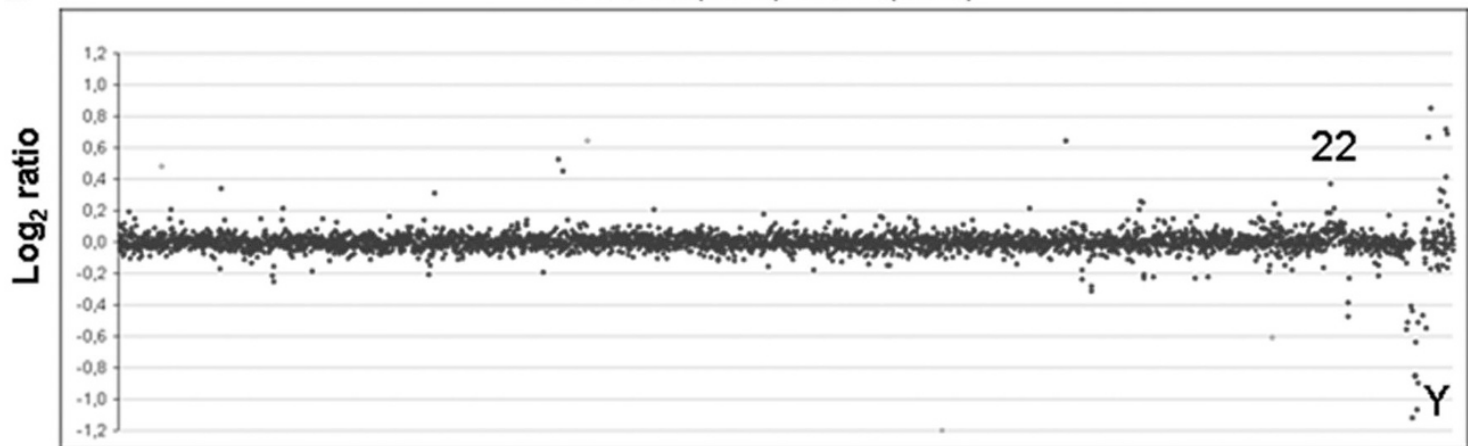

Fig. 1. Array $\mathrm{CGH}$ profiles using DNA from a mosaic monosomy $X(A)$, a male $P O C$ with maternal contamination (B), a male mosaic for an isochromosome $7 p(C)$, and a low-grade mosaicism of chromosome 22 (D) versus DNA from an XXY cell line. For each panel, the $x$-axis represents the clones ordered from the short-arm telomere to the long-arm telomere and chromosomes are ordered from chromosomes 1 to $22, X$ and $Y$. The $y$-axis shows $\log _{2}$ transformed intensity ratios of the combined dye-swap experiments at each locus (patient Cy5/control Cy3). The chromosomes that are not present in equal ratios are indicated. 

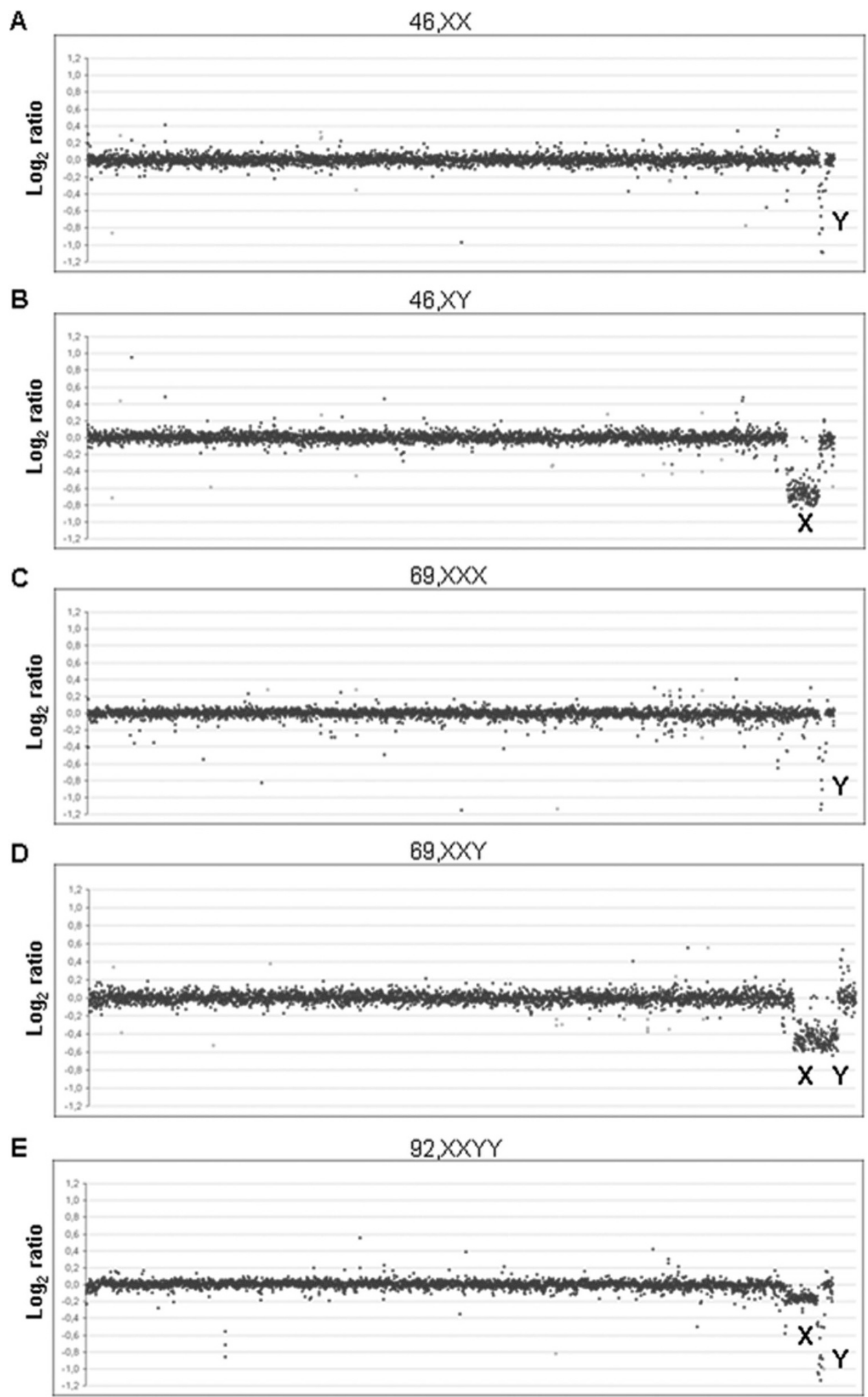

Fig. 2. Array CGH profiles using DNA from a normal female fetus (A), a normal male fetus (B), an $X X X$ triploid fetus (C), an XXY triploid fetus (D), and an XXYY tetraploid fetus (E) versus DNA from an XXY cell line. For each panel, the $X$-axis represents the clones ordered from the short-arm telomere to the long-arm telomere and chromosomes are ordered from chromosomes 1 to $22, X$ and $Y$. The $y$-axis shows $\log _{2}$ transformed intensity ratios of the combined dye-swap experiments at each locus (patient Cy5/control Cy3). The chromosomes that are not present in equal ratios are indicated. 
Removal of unincorporated dNTP's and other components was carried out with the BioPrime ${ }^{\circledR}$ Array CGH Purification Module (Invitrogen, Sunnydale, CA). After labeling, a larger amount of DNA was present. A total of $1750 \mathrm{ng}$ each of labeled patient and reference DNA were combined with $50 \mu \mathrm{g}$ Cot-1 DNA, followed by an ethanol precipitation and resuspension of the pellet in $17 \mu \mathrm{L}$ hybridization buffer. ${ }^{25}$ Blocking solution was made with $16.6 \mu \mathrm{g}$ Cot-1 DNA and $100 \mu \mathrm{g}$ salmon sperm DNA in 20 $\mu \mathrm{L}$ hybridization buffer. Blocking and hybridization were performed as previously described by Vermeesch et al. ${ }^{25}$ Signal intensities were measured with the GenePix 4000B scanner (Axon Instruments) and spots were analyzed with GenePix Pro 5.0 imaging software. ${ }^{25}$ All further data analysis was performed with Excel (Microsoft Inc.; Diegem, Belgium). Spot intensities were corrected for local background, and only spots with signal intensities at least 2-fold above background signal intensities were included in the analysis. For each clone, a ratio of Cy5 to $\mathrm{Cy} 3$ fluorescent intensity was calculated. Normalization of the data was achieved by dividing the fluorescent intensity ratio of each spot by the mean of the ratios of the autosomes. Finally, the normalized ratio values of the duplicates were averaged and a $\log _{2}$ value was calculated. Results of the dye-swap experiments were combined to further reduce the standard deviation. ${ }^{25}$ The lower threshold for positive loci was defined as $4 \mathrm{x}$ the standard deviation of the intensity ratios of all loci. The upper threshold was the empirically set value of 0.40 for the $\log _{2}$ of the intensity ratios. Abnormalities were reported when more than five consecutive clones showed an aberrant ratio.

\section{FISH analysis}

Any losses or gains identified by array CGH without corresponding karyotype were verified using FISH with commercial probes. FISH was performed as described ${ }^{26}$ using an optical fluorescence microscope (Olympus U-SPT, BX51, Japan) with selective filters equipped with CytoVision ${ }^{\circledR}$ software (Applied Imaging, Genetix, Gateshead, UK). A minimum of 100 interphase nuclei were analyzed per sample.

\section{RESULTS}

A total of 103 spontaneous abortion samples were collected for analysis by conventional cytogenetics and array CGH. In 26 cases $(25 \%)$, T-banding analysis could not be performed as a result of culture failure. Twelve samples (12\%) yielded insufficient DNA amounts for array CGH analysis. For five of these, both tissue culture and DNA extraction failed (Table 1).

Thirty-seven samples had a normal female karyotype, whereas only four samples had a normal male karyotype in both techniques. This points to a high level of maternal cell contamination. Any 46,XX results obtained by karyotyping or array $\mathrm{CGH}$ could, therefore, be falsely categorized as normal. Array CGH proved that seven samples identified as having a normal female karyotype by T-banding were either a mixture of female (maternal) with male cells (Fig. 1B), or carried another chromosomal anomaly in only a fraction of the cells (Fig. 1D). For all but four samples $(21,23,33,65)$, the abnormalities identified by array $\mathrm{CGH}$ that were not concordant with the karyotype or where mosaicism or maternal cell contamination was suspected are FISH confirmed.

T-banding detected 22 numerical chromosome aberrations including six monosomies $\mathrm{X}$, two trisomies 13, 16, 21, and 22, one trisomy 8 and 15, four triploidies, one tetraploidy (92,XXYY), and one marker chromosome.

Twenty-six numerical chromosome aberrations were found by array CGH: eight monosomies $\mathrm{X}$, four trisomies 21 and 22, two trisomies 13,16 and $\mathrm{X}$, one trisomy 8 and 15 , and one monosomy 5 .

Three of the monosomy X cases were only detected by array $\mathrm{CGH}$. The average intensity ratio of the $\mathrm{X}$-derived clones was between 0 and -1 , suggesting the presence of normal maternal cells (Fig. 1A). Six trisomies $(13,2 \times 21,2 \times 22, \mathrm{XXX})$ and a monosomy 5 identified by array $\mathrm{CGH}$ were not identified by conventional cytogenetics due to culture failure. In contrast, a trisomy 13 and a monosomy $\mathrm{X}$ identified by conventional cytogenetics were not identified by arrays because of the low DNA concentration.

Two reciprocal translocations identified by conventional cytogenetic analysis were not detected on array CGH, suggesting they are balanced translocations. One sample revealed a deletion of five clones $(787.5 \mathrm{~kb})$ at the steroid sulfatase locus. The deletion was confirmed by FISH. Another structural aberration was an isochromosome of 7p. T-banding analysis of this POC sample showed $100 \% 46, \mathrm{XY}, \mathrm{i}(7 \mathrm{p})$ cells. The array results, however, identified the presence of one $\mathrm{X}$ chromosome, but indicated that the duplication of $7 p$ and the deletion of $7 q$ were only present in some instead of all cells (Fig. 1C), suggesting mosaicism of the isochromosome $7 \mathrm{p}$ in this male patient. FISH analysis verified these array results by identifying two distinct cell populations: one with $46, X Y$ cells and one with $46, X Y, i(7 p)$ cells.

\section{DISCUSSION}

Cytogenetic abnormalities have been shown to account for about $50 \%$ of spontaneous abortions, with autosomal trisomies and monosomy $\mathrm{X}$ being the most common findings. In this study, the overall abnormality rate was $36 \%$ (35 of 96). Neither technique was able to identify all aberrations, with T-banding detecting 63\% (22 of 35) and array CGH 83\% (29 of 35). Of the 70 samples that were successfully analyzed by both techniques, 55 (79\%) revealed identical karyotypes (42 normal, 13 abnormal) and 15 (21\%) cases showed discrepancies.

Several studies using $\mathrm{CGH}$ or array $\mathrm{CGH}$ to analyze spontaneous abortions have been published.4,5,13-17,20-22,24 Abnormalities were detected in anywhere from $38 \%$ to $72 \%$ of samples. This wide range was caused by differences in study size, absence of a second technique to detect polyploidies, or use of samples that failed to grow. In case a comparison with conventional cytogenetics was made, results were usually concordant. Array CGH mostly failed to detect triploidies, but identified a number of smaller deletions and duplications and showed that some 46,XX samples were in fact abnormal but contaminated by maternal cells. Our study presented the same characteristics, but had a high percentage of maternal cell contamination leading to a lower overall abnormality rate $(36 \%)$. This contamination was responsible for the main difference in results between conventional and molecular karyotyping. There are likely two reasons why this contamination has a larger effect on conventional karyotyping: (1) During the cell culture, selection against chromosomal abnormal cells may occur ${ }^{27}$ (2) Array CGH is better suited to detect low-grade mosaicism. ${ }^{27}$

Array CGH analysis of spontaneous miscarriages is advantageous compared with traditional cytogenetics. First, no cell culture is required. This avoids culture failure, culture contamination, overgrowth with maternal cells, or selection against the chromosomal abnormal cells of mosaic fetuses. The influence of maternal cell overgrowth can be seen in the slightly larger male to female ratio for array CGH (17 of 100) compared to T-banding (10 of 100) among cases identified as normals. However, both these ratios still show a serious sex ratio bias. These findings indicate that samples not only suffer from maternal cell overgrowth 
during tissue culture, as seen in the study by Bell et al., ${ }^{4}$ but that villi samples are also often contaminated with maternal cells and that it remains difficult to take an accurate sample of fetal material. Therefore, 46, XX results should always be reported with caution.

An added advantage of the elimination of tissue culture is the reduced turn around time for cytogenetic analysis of POC. With the prospect of further automation of the array CGH process, this will only become shorter.

Previous studies have shown the ability of array CGH with BAC arrays to detect low-grade mosaicism, putting a lower threshold around $10 \% .^{25,28,29}$ The lowest grade mosaic described is $7 \%$ monosomy 7.27 In this study, we identified three cases with a mosaicism level of $10 \%$ to $20 \%$ : a $46, \mathrm{XY}$ sample mosaic for an isochromosome 7p (Fig. 1C), another male with 20\% trisomy 13 , and a female case with $10 \%$ trisomy 22 (Fig. 1D). In addition, five cases were diagnosed as normal female karyotypes by conventional cytogenetics, but were later confirmed by FISH to be either monosomy X (Turner) (Fig. 1A) or normal males (Fig. 1B). On array, these two types of anomalies both showed an intermediate $\log _{2}$ value for the sex chromosomes, but they could be distinguished from one another, specifically at the Y chromosome. In Turner patients, the $\mathrm{Y}$ chromosome was missing, with $\log _{2}$ ratios of -2 to -4 . Normal $X Y$ cases contaminated with maternal cells showed only partially lowered $\mathrm{Y}$ values, with $\log _{2}$ ratios ranging from -0.6 to -1.3 . Using this method, we identified cases of maternal contamination with as low as 5 to $7 \% 46, \mathrm{XY}$ cells.

The array CGH technique was able to detect all trisomies and monosomies previously identified by T-banding. By using 47, XXY reference DNA, the difference between sex chromosome abnormalities such as trisomies and tetrasomies of the $\mathrm{X}$ chromosome and normal 46,XX and 46,XY cases was more readily observed, confirming previous results by Ballif et al. ${ }^{24}$ Some triploidies (XXY, not XXX) (Fig. 2, C and D) and some tetraploidies (all XXYY) (Fig. 2E) could also be detected. The tetraploidies created a pattern similar to that of a male partially masked by maternal contamination (Fig. 1B). Despite the observations made in these few cases, polyploidy and balanced translocations are usually not detectable by metaphase- or arraybased CGH. Other groups have dealt with this by performing flow cytometry assays ${ }^{5}$ for polyploidy. We now apply interphase FISH using the $\mathrm{X}$ and $\mathrm{Y}$ probes on uncultured fetal tissue to identify potential triploidies or tetraploidies. Subsequently array CGH is performed. This combination allows all chromosomal imbalances as well as tri- and tetraploidies to be identified.

Array CGH has a higher resolution than conventional karyotyping, making it possible to detect small deletions and duplications, as seen in the detection of the steroid sulfatase deletion described earlier. The resolution of recently developed arrays has been steadily increasing and is only limited by the size of the clones and the distance between clones. This raises the opportunity to detect even smaller deletions and duplications, which will identify new regions or genes that play a role in early embryonic development. Increased resolution arrays come, however, at a higher cost. Currently, the cost of performing lowresolution array $\mathrm{CGH}$ is comparable with the cost of karyotyping.

Overall this study shows that the use of array CGH for the cytogenetic evaluation of POC is an improvement compared with classical cytogenetics.

\section{ACKNOWLEDGMENTS}

This work was made possible by Grants from the IWT (SBO60848), FWO, GOA/2006/12, and Center of Excellence SymBioSys (Research Council K.U.Leuven EF/05/007) (J.R.V.).
We are also grateful to the Mapping Core and Map finishing groups of the Wellcome Trust Sanger Institute for initial BACclone supply and verification and the microarray facility, Flanders Interuniversity Institute for Biotechnology (VIB), for their help in spotting the arrays.

\section{REFERENCES}

1. Hassold T, Chen N, Funkhouser J, et al. A cytogenetic study of 1000 spontaneous abortions. Ann Hum Genet 1980;44:151-178.

2. Hassold T. A cytogenetic study of repeated spontaneous abortions. Am J Hum Genet 1980;32:723-730.

3. Goddijn M, Leschot N. Genetic aspects of miscarriage. Baillieres Best Pract Res Clin Obstet Gynaecol 2000;14:855-865.

4. Bell K, Van Deerlin P, Haddad B, Feinberg R. Cytogenetic diagnosis of "normal 46,XX" karyotypes in spontaneous abortions frequently may be misleading. Fertil Steril 1999;71:334-341.

5. Lomax B, Tang S, Separovic E, et al. Comparative genomic hybridization in combination with flow cytometry improves results of cytogenetic analysis of spontaneous abortions. Am J Hum Genet 2000;66:1516-1521.

6. Bruno DL, Burgess T, Ren H, et al. High-throughput analysis of chromosome abnormality in spontaneous miscarriage using an MLPA subtelomere assay with an ancillary FISH test for polyploidy. Am J Med Genet A 2006; 140:2786-2793.

7. Diego-Alvarez D, Garcia-Hoyos M, Trujillo MJ, et al. Application of quantitative fluorescent PCR with short tandem repeat markers to the study of aneuploidies in spontaneous miscarriages. Hum Reprod 2005; 20:1235-1243.

8. Jobanputra V, Sobrino A, Kinney A, Kline J, Warburton D. Multiplex interphase FISH as a screen for common aneuploidies in spontaneous abortions. Hum Reprod 2002;17:1166-1170.

9. Mann K, Fox SP, Abbs SJ, et al. Development and implementation of a new rapid aneuploidy diagnostic service within the UK National Health Service and implications for the future of prenatal diagnosis. Lancet 2001;358:1057-1061.

10. Kallioniemi A, Kallioniemi O, Sudar D, et al. Comparative genomic hybridization for molecular cytogenetic analysis of solid tumors. Science 1992; 258:818-821.

11. Kirchhoff M, Rose H, Lundsteen C. High resolution comparative genomic hybridisation in clinical cytogenetics. J Med Genet 2001;38:740-744.

12. Daniely M, Barkai G, Goldman B, Aviram-Goldring A. Detection of numerical chromosome aberrations by comparative genomic hybridization. Prenat Diagn 1999;19:100-104.

13. Barrett I, Lomax B, Loukianova T, Tang S, Lestou V, Kalousek D. Comparative genomic hybridization: a new tool for reproductive pathology. Arch Pathol Lab Med 2001;125:81-84.

14. Hu Y, Chen X, Chen L, Xu Z, Wang X, Cui H. Comparative genomic hybridization analysis of spontaneous abortion. Int J Gynaecol Obstet 2006; 92:52-57.

15. Azmanov D, Milachich T, Zaharieva B, et al. Profile of chromosomal aberrations in different gestational age spontaneous abortions detected by comparative genomic hybridization. Eur J Obstet Gynecol Reprod Biol 2007;131:127-131

16. Fritz B, Hallermann C, Olert J, et al. Cytogenetic analyses of culture failures by comparative genomic hybridisation (CGH)-re-evaluation of chromosome aberration rates in early spontaneous abortions. Eur J Hum Genet 2001;9: 539-547.

17. Tabet A, Aboura A, Dauge M, et al. Cytogenetic analysis of trophoblasts by comparative genomic hybridization in embryo-fetal development anomalies. Prenat Diagn 2001;21:613-618.

18. Pinkel D, Segraves R, Sudar D, et al. High resolution analysis of DNA copy number variation using comparative genomic hybridization to microarrays. Nat Genet 1998;20:207-211.

19. Snijders A, Nowak N, Segraves R, et al. Assembly of microarrays for genomewide measurement of DNA copy number. Nat Genet 2001;29:263-264.

20. Schaeffer A, Chung J, Heretis K, Wong A, Ledbetter D, Lese Martin C. Comparative genomic hybridization-array analysis enhances the detection of aneuploidies and submicroscopic imbalances in spontaneous miscarriages. Am J Hum Genet 2004;74:1168-1174.

21. Benkhalifa M, Kasakyan S, Clement P, et al. Array comparative genomic hybridization profiling of first-trimester spontaneous abortions that fail to grow in vitro. Prenat Diagn 2005;25:894-900.

22. Shimokawa O, Harada N, Miyake N, et al. Array comparative genomic hybridization analysis in first-trimester spontaneous abortions with 'normal' karyotypes. Am J Med Genet A 2006;140:1931-1935.

23. Dutrillaux B. New system of chromosome banding: the T bands. Chromosoma 1973;41:395-402.

24. Ballif B, Kashork C, Saleki R, et al. Detecting sex chromosome anomalies and common triploidies in products of conception by array-based comparative genomic hybridisation. Prenat Diagn 2006;26:333-339. 
25. Vermeesch J, Melotte C, Froyen G, et al. Molecular karyotyping: array CGH quality criteria for constitutional genetic diagnosis. J Histochem Cytochem 2005;53:413-422.

26. Vermeesch J, Mertens G, David G, Marynen P. Assignment of the human glypican gene (GPC1) to $2 \mathrm{q} 35-\mathrm{q} 37$ by fluorescence in situ hybridization. Genomics 1995;25:327-329.

27. Menten B, Maas N, Thienpont B, et al. Emerging patterns of cryptic chromosomal imbalance in patients with idiopathic mental retardation and multiple congenital anomalies: a new series of 140 patients and review of published reports. J Med Genet 2006;43:625-633.

28. Ballif B, Rorem E, Sundin K, et al. Detection of low-level mosaicism by array CGH in routine diagnostic specimens. Am J Med Genet A 2006;140: 2757-2767.

29. Cheung S, Shaw C, Scott D, et al. Microarray-based CGH detects chromosomal mosaicism not revealed by conventional cytogenetics. Am J Med Genet A 2007; 143:1679-1686. 